\title{
Gravitational instability and the formation of giant planets by core accretion
}

\author{
K. Kornet ${ }^{1}$, S. Wolf ${ }^{1}$ and M. Różyczka ${ }^{2}$ \\ ${ }^{1}$ Max Planck Institute for Astronomy, Königstuhl 17, 69117 Heidelberg, Germany \\ email: \{kornet,swolf\}@mpia-hd.mpg.de \\ ${ }^{2}$ Nicolaus Copernicus Astronomical Center, Bartycka 18, Warsaw, 00-716, Poland \\ email:mnr@camk.edu.pl
}

\begin{abstract}
We investigate the problem of giant planet formation around stars with various masses based on the core accretion/gas capture model. At first, we follow the evolution of gas and solids from the moment when all solids are in the form of small grains to the stage when most of them have reached planetesimal size. We show that the surface density of a planetesimal swarm tends to be higher around less massive stars. Subsequently, we derive the minimum surface density of the planetesimal swarm required for the formation of giant planets, both in a numerical and in an approximate analytical approach. We combine these results by calculating a set of representative disk models, characterized by different masses, sizes, and metallicities. This allows us to quantify the probability of each individual disk model to form giant planets. Furthermore, we take the fact into account, that in some of these models, the outer regions of the disks become gravitationally unstable.
\end{abstract}

Keywords. protoplanetary disk, planetary systems: formation

\section{Introduction}

Radial velocity surveys led to discovery of over 150 extrasolar planets around main sequence stars Marcy et al. (2005), Mayor et al. (2004). Those surveys have been most successful in the case of $\mathrm{G}$ dwarf stars. Due to the constant progress in the detection techniques, the observational programs recently started to include also stars with lower masses on a larger scale, namely $\mathrm{M}$ dwarfs. Moreover, some of these surveys are now particularly dedicated to lower-mass stars Endl et al. (2003), Bonfils et al. (2004).

From the theoretical point of view the problem of giant planet formation around $\mathrm{M}$ dwarfs was studied recently by Laughlin et al. (2004). They addressed it within the core accretion - gas capture model (CAGCM). They conclude that $\mathrm{M}$ dwarfs have a limited ability to form Jupiter-mass planets. This is a direct consequence of their assumption that the surface density of the planetesimal swarm out of which planetary cores are formed scales linearly with the mass of the central star. However, the solid component of the protoplanetary disk evolves in a different way than the gaseous component Weidenschilling \& Cuzzi (1993). Due to the gas drag a significant redistribution of solids takes place, and in the inner disk their surface density can be substantially enhanced compared to the initial one Weidenschilling (2003), Stepinski \& Valageas (1997). An obvious conclusion is that the analysis of the formation of giant planets around stars with various masses should include the global evolution of solids in protoplanetary disks.

\section{Methods of calculation}

We model the protoplanetary disk as a two component fluid, consisting of gas and solids. The gaseous component is described as an alpha disk by the analytical model of 
Stepinski (1998) which describes its properties, as a function of distance $a$ from the star and time $t$, in terms of a self-similar solution to the viscous diffusion equation.

The main assumptions underlying our approach to the evolution of solids are (1) at each radial distance from the central star the particles have the same size (which in general varies over time), (2) there is only one component of dust, in this case corresponding to high-temperature silicates with the evaporation temperature $T_{\text {evap }}=1350 \mathrm{~K}$ and bulk density $3.3 \mathrm{~g} \mathrm{~cm}^{-3}$, (3) all collisions between particles lead to coagulation. At each radius, the vertical extent of the solid particle distribution is calculated and is evolved in time, so the effect of sedimentation of solids toward the midplane of the disk is taken into account. All assumptions and approximations are discussed in Stepinski \& Valageas (1997), Stepinski (1998) and Kornet et al. (2001).

We model the formation of a giant planet in situ, so the orbital parameters of the planet do not vary in time. Our procedure is based on the following assumptions: (1) core accretion starts when solids at a given distance $a$ from the star reach radii of $2 \mathrm{~km}$, (2) at each time, the planetesimals are well mixed through the feeding zone of the planet; thus, their surface density $\Sigma_{\mathrm{s}}$ is always uniform in space, but usually decreasing with time as planetesimals accrete onto the planet, (3) the planetesimals do not migrate into the feeding zone from outside or inside, and vice versa, but they can be overtaken by the boundary of the feeding zone as it expands due to the growing mass of the planet, (4) to calculate the rate of gas accretion onto the planet, following Ida \& Lin (2004a), we use a simplified approach based on fits to the numerical results Bryden et al. (2000).

\section{Results}

In the CAGCM the process of planet formation is naturally split in two main phases. In the first one a planetesimal swarm is formed in the protoplanetary disk. In the second phase planetary cores are assembled which subsequently accrete planetesimals and gas from the disk.

To illustrate how the mass of the central star influences the formation of the planetesimal swarm, we follow the evolution of a protoplanetary disk with an initial mass $M_{0}=0.1 M_{\odot}$ and initial outer radius $R_{0}=1000 \mathrm{AU}$ for three values of $M_{\star}(0.5,1$ and $\left.4 M_{\odot}\right)$. Initially, the dust is well mixed with the gas, with the ratio $\Sigma_{\mathrm{s}} / \Sigma_{\mathrm{g}}=6 \times 10^{-3}$ being constant everywhere in the disk. As the disk evolves the grains grow in size due to mutual collisions and gain inward radial velocities due to the gas drag. If their growth time of is shorter than the timescale of inward migration, they manage to reach sizes of a few $\mathrm{km}$ before reaching the evaporation radius. Their radial motions are then stopped and the planetesimal swarm attains its final form. Fig. 1 shows the distribution of planetesimals in our model after $10^{6} \mathrm{yr}$ from the beginning of its evolution. In all three cases, the outer radius of the planetesimal swarm is much smaller than the initial outer radius of the disk. The difference is larger for models with smaller $M_{\star}$. This is because small solid bodies evolving in disks around less massive stars gain larger inward velocities and tend to migrate to smaller radii before reaching kilometer sizes. Due to the inward migration of solids and their confinement to much smaller radii, the final surface density of planetesimals is increased locally by factor of a few in comparison with the initial value of $\Sigma_{\mathrm{s}}$. This effect is larger in disks around less massive stars.

To quantify the influence of $M_{\star}$ on the effectiveness of giant planet formation from a planetesimal swarm, we introduce the concept of the minimum surface density $\Sigma_{\mathrm{s}, \min }$. We define it as the minimum value of the initial surface density of planetesimals $\Sigma_{\mathrm{s} \text {,init }}$ needed to from a Jupiter-mass $\left(1 \mathrm{M}_{\mathrm{J}}\right)$ planet in less than the lifetime of the protoplanetary disk $\tau_{\mathrm{f}}$. For $\tau_{\mathrm{f}}$ we adopt a value of $3 \times 10^{6} \mathrm{yr}$. Fig. 2 shows $\Sigma_{\mathrm{s} \text {,init }}$ as function of distance 


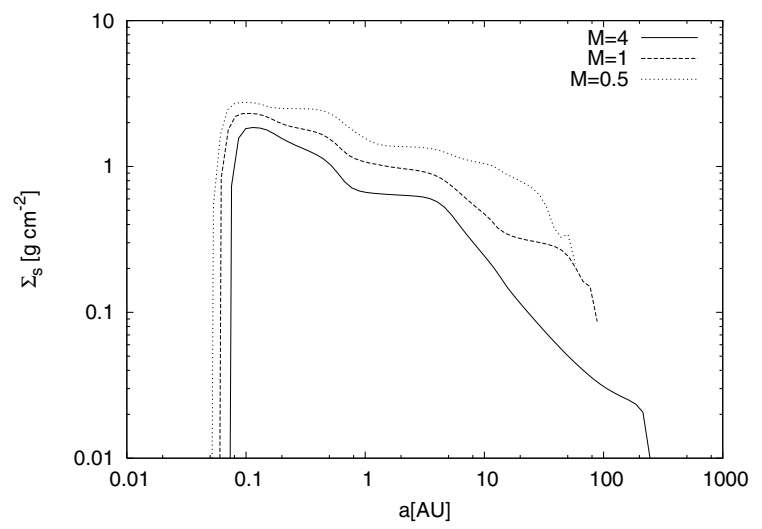

Figure 1. The surface density of solids as a function of the distance from the star in a protoplanetary disk with an initial mass $0.1 M_{\odot}$ and outer radius $1000 \mathrm{AU}$ after $10^{6} \mathrm{yr}$ from the beginning of its evolution. Different curves correspond to different values of the mass of the central star in solar masses as described by the labels.

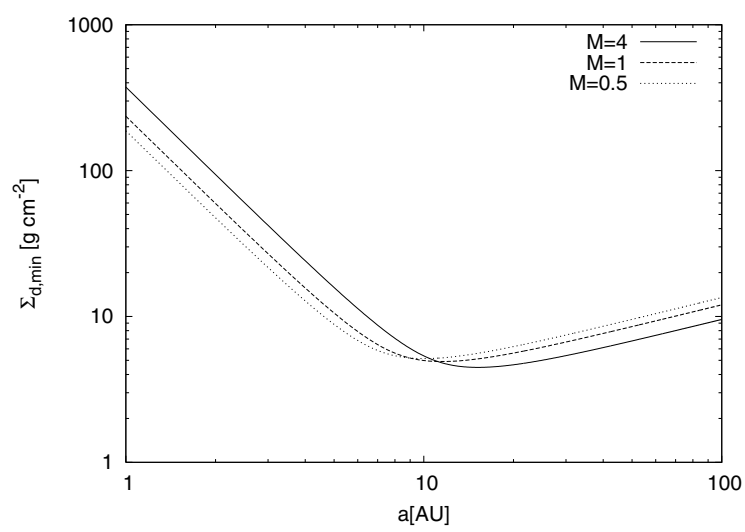

Figure 2. The minimum surface density of a planetesimal swarm needed to from a $1 M_{\mathrm{J}}$ planet in less than $3 \times 10^{6} \mathrm{yr}$ as a function of distance from the central star. Different curves are obtained for different masses of the central star, as labeled in units of solar masses in the upper right corner.

from the star, for the same values of $M_{\star}$ as before. Close to the star $(a<10 \mathrm{AU})$, $\Sigma_{\mathrm{s}, \text { init }} \sim a^{-2}$, so it is a decreasing function of the radius $a$. For sufficiently large radii $(a>10 \mathrm{AU}), \Sigma_{\mathrm{s}, \min }(a)$ changes its slope and becomes an increasing function of $a$. In this regime $\Sigma_{\mathrm{s}, \text { init }} \sim a^{1 / 2}$. We also obtained the approximate, analytical formulas for $\Sigma_{\mathrm{s} \text {,init }}$, which are in a good agreement with the results of numerical calculations.

To investigate the influence of the mass of the central star on the whole process of giant planet formation we calculate a grid of models characterized by different values of the initial disk mass $M_{0}$ and outer radius $R_{0}$. We choose $M_{0}$ in the range between 0.02 and $0.2 M_{\odot}$. The range of $R_{0}$ is adjusted for every metallicity so that all models in which the formation of giant planets is possible is accounted for.

We follow the evolution of each model until all solids are in the form of planetesimals or are accreted onto the star. Then, we evaluate every model with planetesimals to determine whether the surface density of planetesimals exceeds the minimum surface density $\Sigma_{\mathrm{s}, \min }$ anywhere in the disk. Models with this property are labeled as planet bearing. For every such model we determine the minimum and maximum distance from the star at which 

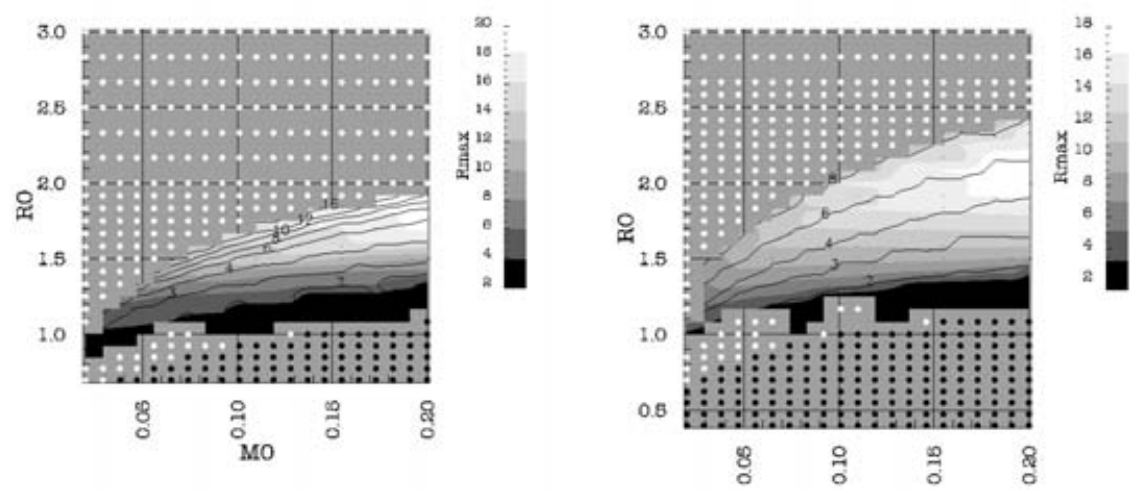

Figure 3. The plane of initial parameters of protoplanetary disk models $\left[M_{0}, \log R_{0}\right]$. Minimum and maximum distance from a $1 M_{\odot}$ (left panel) and $0.5 M_{\odot}$ (right panel) star at which the formation of a $1 M_{\mathrm{J}}$ planet is possible within $3 \times 10^{6} \mathrm{yr}$ is indicated by contours and grey scale, respectively. White circles indicate disk models in which the surface density of the planetesimal swarm is everywhere lower than the critical value for planet formation. Black circles indicate disks in which all solids are accreted onto the star.

the surface density of the planetesimal swarm is larger than $\Sigma_{\mathrm{s} \text {, min }}$. The results obtained for different values of the stellar mass are shown in Fig. 3.

The area of the region occupied on the $\left[M_{0}, R_{0}\right]$ plane by the planet bearing disks is clearly anticorrelated with the mass of the central star. Solid grains gain higher inward velocities in disks around less massive stars, and the resulting planetesimal swarms have higher surface densities. We see that, reducing the mass of the central star increases the maximum $R_{0}$ for which planet formation is possible in disks with the same initial mass $M_{0}$. We can also see that in a given disk the inner edge of the planet-bearing region moves inward as we decrease the mass of the star. This effect is mainly caused by the larger surface density of planetesimal swarms produced by disks around less massive stars. Differences in $\Sigma_{\mathrm{s} \text {,min }}$, while appreciable, are much less important. The outer edge of the planet-bearing region also moves closer to the star. This is because in most cases it coincides with the outer edge of the planetesimal swarm which is more compact around less massive stars. The difference in the minimum surface density $\Sigma_{\mathrm{s} \text {, min }}$ also tends to decrease the outer radius of the planet bearing region, but it is again a second order factor.

One of the main results of extrasolar planet searches is the discovery that planet bearing stars tend to have higher metallicities than field stars Santos et al. (2000), Fischer \& Valenti (2003). Ida \& Lin (2004b) and Kornet et al. (2005) showed that this correlation can be quantitatively reproduced within CAGCM. Herein we extend calculations of Kornet et al. (2005) onto disks around stars with various masses. The change in the disk metallicity influences the processes leading to the formation of planets in two ways. Firstly, it changes the structure of the gaseous disk by changing the opacity in the disk. In our models we scaled the opacity by a constant factor $Z$ equal to the metallicity of the disk expressed in solar units. Secondly, the primordial metallicity of the disk determines the initial ratio of dust to gas surface densities. In our models this ratio is initially independent of the distance from the star and is equal to $\Sigma_{\mathrm{s}} / \Sigma_{\mathrm{g}}=6 \times 10^{-3} Z$. We compute grids of models similar to those described above for eight different values of $Z$ distributed between 0.2 and 3. Additionally, for every value of $\left[M_{0}, R_{0}\right]$ we check the gravitational stability of the corresponding gaseous disk. In some cases the value of the Toomre parameter $Q$ drops below 1 in the outer region of the disk. We assume that such region 


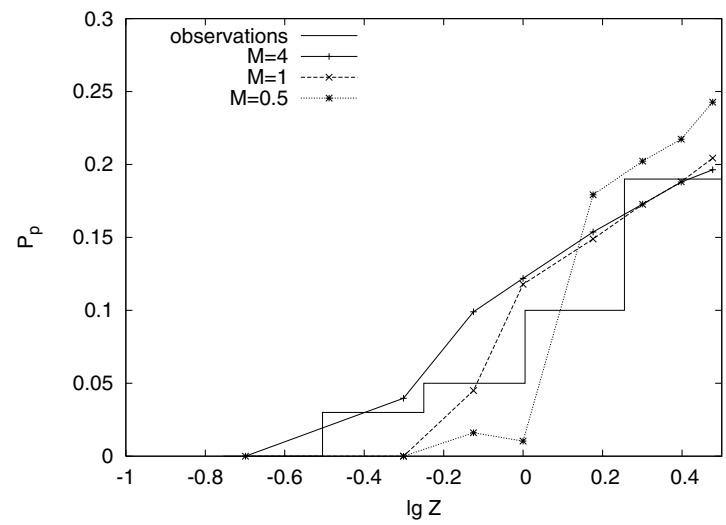

Figure 4. The rate of planet occurrence as a function of the primordial metallicity of protoplanetary disks. Different lines are obtained for models with different masses of the central star, as labeled in solar units in the upper left corner. The histogram shows the observational data compiled by Fischer \& Valenti (2003).

fragments and some giant planets are formed there on a very short time scale. In that case, we use modified initial values of $M_{0}$ and $R_{0}$, which correspond to the mass and outer radius of the stable part of the original disk. Following the procedure described by Kornet et al. (2005), for every $Z$ we calculate the area $A_{\mathrm{p}, 5}$ of the region occupied on the $\left[M_{0}, \log R_{0}\right]$ plane by disks that form planets at distances smaller than $5 \mathrm{AU}$ from the central star. A measure of the rate of planet occurrence can be defined as $P_{\mathrm{p}}=A_{\mathrm{p}, 5} / C$, where the normalization factor $C$ is chosen in such a way as to reproduce the observed value of $P_{\mathrm{p}}$ for $Z=3$ and $M_{\star}=1 M_{\odot}$.

The results are presented on Fig. 4 , showing the rate of planet occurrence as a function of disk metallicity for three values of $M_{\star}$. As expected, $P_{\mathrm{p}}$ is an increasing function of $Z$. The minimum value of $Z$ below which no giant planets are formed at orbits smaller than $5 \mathrm{AU}$ decreases with the mass of the central star from $Z_{\min } \approx 0.6$ for $M_{\star}=0.5 M_{\odot}$, to $Z_{\min } \approx 0.2$ for $M_{\star}=4 M_{\odot}$.

We see that for $Z$ smaller than $\sim 0.2, P_{\mathrm{p}}$ is an increasing function of $M_{\star}$. This is because most disks in which formation of planets would be possible around less massive stars have outer parts which are gravitationally unstable, and the amount of solids present in their stable parts is too low to enable subsequent formation of giant planets according to the CAGCM. However, as $Z$ increases, smaller and smaller disks become planetbearing for every $M_{0}$, and the percentage of gravitationally unstable disks in which formation of giant planets is not possible decreases. Consequently, the factors promoting the formation of giant planets around less massive stars as described in previous sections become important, and $P_{\mathrm{p}}$ changes into a decreasing function of $M_{\star}$.

\section{Discussion}

Obviously, our description of the evolution of solids is very simplified. The basic underlying assumptions like the single-size distribution of solid grains or the neglect of planet migration were already discussed by Kornet et al. (2004) and Kornet et al. (2005). The main additional assumption introduced in the present paper is the independence of the initial parameters of protoplanetary disks on the mass of the central star. While admittedly ad hoc, it seems to be better than that adopted by Laughlin et al. (2004), who scaled their initial surface density of planetesimals linearly with the mass of the star. They did 
not take into account the antecedent evolution of solids leading to the formation of planetesimal swarms, and concluded that the formation of giant planets around low-mass stars is difficult. Our models show that it is a vital factor facilitating the formation of giant planets, whose role should be particularly well seen for low-mass stars.

\section{Acknowledgements}

This project was supported by the German Research Foundation (DFG) through the Emmy Noether grant WO 857/2-1 and the European Community's Human Potential Programme trough the contract HPRN-CT-2002-00308, PLANETS. MR acknowledges the support from the grant No. 1 P03D 02626 from the Polish Ministry of Science.

\section{References}

Bonfils, X., Delfosse, X., Udry, S., et al. 2004, in Astronomical Society of the Pacific Conference Series, 101

Bryden, G., Lin, D. N. C., \& Ida, S. 2000, ApJ, 544, 481

Endl, M., Cochran, W. D., Tull, R. G., \& MacQueen, P. J. 2003, AJ, 126, 3099

Fischer, D. \& Valenti, J. A. 2003, in Scientific Frontiers in Research on Extrasolar Planets, ASP Conference Series, Vol 294, ed. D. Deming \& S. Seager (San Francisco: ASP), 117

Ida, S. \& Lin, D. N. C. 2004a, ApJ, 604, 388

Ida, S. \& Lin, D. N. C. 2004b, ApJ, 616, 567

Kornet, K., Bodenheimer, P., Różyczka, M., \& Stepinski, T. F. 2005, A\&A, 430, 1133

Kornet, K., Stepinski, T. F., \& Różyczka, M. 2001, A\&A, 378, 180

Kornet, K., Stepinski, T. F., \& Różyczka, M. 2004, A\&A, 417, 151

Laughlin, G., Bodenheimer, P., \& Adams, F. C. 2004, ApJ, 612, L73

Marcy, G. W., Butler, R. P., Vogt, S. S., et al. 2005, ApJ, 619, 570

Mayor, M., Udry, S., Naef, D., et al. 2004, A\&A, 415, 391

Santos, N. C., Israelian, G., \& Mayor, M. 2000, A\&A, 363, 228

Stepinski, T. F. 1998, Icarus, 132, 100

Stepinski, T. F. \& Valageas, P. 1997, A\&A, 319, 1007

Weidenschilling, S. J. 2003, in Lunar and Planetary Science Conference \#34, abstract \#1707

Weidenschilling, S. J. \& Cuzzi, J. N. 1993, in Protostars and Planets III, ed. E. Levy \& J. Lunine (Tuscon: Univ. of Arizona Press), 1031 